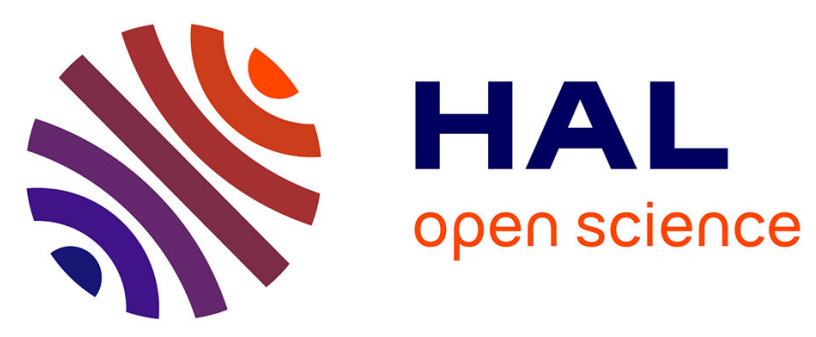

\title{
Overcoming power broadening of the quantum dot emission in a pure wurtzite nanowire
}

D W Reimer, V Bulgarini, D Fognini, Reinier W. Heeres, J J Witek, V a M Versteegh, D Rubino, P W Braun, P W Kamp, P W Höfling, et al.

\section{- To cite this version:}

D W Reimer, V Bulgarini, D Fognini, Reinier W. Heeres, J J Witek, et al.. Overcoming power broadening of the quantum dot emission in a pure wurtzite nanowire. Physical Review B: Condensed Matter and Materials Physics (1998-2015), 2016, 93, pp.195316. 10.1103/PhysRevB.93.195316 . cea01485240

HAL Id: cea-01485240

https://hal-cea.archives-ouvertes.fr/cea-01485240

Submitted on 8 Mar 2017

HAL is a multi-disciplinary open access archive for the deposit and dissemination of scientific research documents, whether they are published or not. The documents may come from teaching and research institutions in France or abroad, or from public or private research centers.
L'archive ouverte pluridisciplinaire HAL, est destinée au dépôt et à la diffusion de documents scientifiques de niveau recherche, publiés ou non, émanant des établissements d'enseignement et de recherche français ou étrangers, des laboratoires publics ou privés. 


\title{
Overcoming power broadening of the quantum dot emission in a pure wurtzite nanowire
}

\author{
M. E. Reimer, ${ }^{1,2,}{ }^{*}$ G. Bulgarini, ${ }^{1,3}$ A. Fognini, ${ }^{1}$ R. W. Heeres, ${ }^{1}$ B. J. Witek, ${ }^{1}$ M. A. M. Versteegh, ${ }^{1}$ A. Rubino, ${ }^{1}$ T. Braun, ${ }^{4}$ \\ M. Kamp, ${ }^{4}$ S. Höfling, ${ }^{4,5}$ D. Dalacu, ${ }^{6}$ J. Lapointe, ${ }^{6}$ P. J. Poole, ${ }^{6}$ and V. Zwiller ${ }^{1}$ \\ ${ }^{1}$ Kavli Institute of Nanoscience, Technical University of Delft, Delft, The Netherlands \\ ${ }^{2}$ Institute for Quantum Computing and Department of Electrical \& Computer Engineering, University of Waterloo, Waterloo, Canada \\ ${ }^{3}$ Single Quantum B.V., Delft, The Netherlands \\ ${ }^{4}$ Technische Physik, Physikalisches Institut, Universität Würzburg, Würzburg, Germany \\ ${ }^{5}$ SUPA, School of Physics and Astronomy, University of St Andrews, St Andrews, United Kingdom \\ ${ }^{6}$ National Research Council of Canada, Ottawa, Canada
}

(Received 4 November 2015; revised manuscript received 4 March 2016; published 25 May 2016)

\begin{abstract}
One of the key challenges in developing quantum networks is to generate single photons with high brightness, purity, and long temporal coherence. Semiconductor quantum dots potentially satisfy these requirements; however, due to imperfections in the surrounding material, the coherence generally degrades with increasing excitation power yielding a broader emission spectrum. Here we overcome this power-broadening regime and demonstrate an enhanced coherence at exciton saturation where the detected count rates are highest. We detect single-photon count rates of 460000 counts per second under pulsed laser excitation while maintaining a single-photon purity greater than $99 \%$. Importantly, the enhanced coherence is attained with quantum dots in ultraclean wurtzite InP nanowires, where the surrounding charge traps are filled by exciting above the wurtzite InP nanowire band gap. By raising the excitation intensity, the number of possible charge configurations in the quantum dot environment is reduced, resulting in a narrower emission spectrum. Via Monte Carlo simulations we explain the observed narrowing of the emission spectrum with increasing power. Cooling down the sample to $300 \mathrm{mK}$, we further enhance the single-photon coherence twofold as compared to operation at $4.5 \mathrm{~K}$, resulting in a homogeneous coherence time, $T_{2}$, of $1.2 \mathrm{~ns}$, and two-photon interference visibility as high as $83 \%$ under strong temporal postselection ( $\sim 5 \%$ without temporal postselection).
\end{abstract}

DOI: 10.1103/PhysRevB.93.195316

\section{INTRODUCTION}

A quantum network is constituted by local nodes where quantum information is generated, processed, and stored, as well as communication channels between these nodes to coherently transfer quantum states across the entire network [1]. The messengers of choice to distribute quantum information over long distances are single photons since they interact very weakly with the environment, thereby preserving their coherence, and are compatible with existing telecommunication fiber technologies. Coherent single photons are necessary in future quantum technologies such as linear optics quantum computing [2], quantum teleportation [3], interfacing remote quantum bits [4,5], or integration of a quantum repeater [6]. In addition to coherence, the source brightness and single-photon purity (i.e., suppressed multiphoton emission) are extremely important toward practical implementation of these quantum technologies.

Semiconductor quantum dots embedded in photonic structures are leading candidates to generate coherent and bright sources of single photons with high purity. However, increasing the source brightness typically comes at the cost of degrading the coherence, resulting in a broadened emission spectrum [7-10]. This effect, known as "power broadening," is attributed to an increase in the charge fluctuations of the quantum dot environment that leads to spectral wandering [7]. Here, we report on the single-photon coherence from an ultraclean material system consisting of a single quantum dot

\footnotetext{
*mreimer@uwaterloo.ca
}

in a pure wurtzite InP nanowire, where dephasing mechanisms are suppressed by both cooling and higher excitation power. Remarkably, in stark contrast to conventional self-assembled quantum dots [7-15], we obtain the longest coherence at the highest brightness of the single-photon emission. Importantly, we detect single-photon count rates of 460 kilocounts per second while maintaining a single-photon purity greater than 99\%. This latter feature provides a significant advantage over cavity-based nanostructures where increased multiphoton events occur at saturation of the quantum dot emission due to cavity feeding of other quantum dots or detuned transitions of the same quantum dot $[16,17]$. Finally, by cooling the sample to $300 \mathrm{mK}$ we show that the coherence can be enhanced by a factor of $\sim 2$ as compared to operation at $4.5 \mathrm{~K}$ by further suppressing interactions with phonons. We further analyze the degree of photon indistinguishability in the case of this enhanced coherence and obtain a two-photon visibility as high as $83 \%$ under strong temporal postselection $(\sim 5 \%$ without temporal postselection).

A scanning electron microscope image of a typical InP tapered nanowire waveguide containing a single InAsP quantum dot used in the present experiments is shown in Fig. 1(a). There are three main features to this design in order to achieve both bright single-photon emission and a long temporal coherence. First, a waveguide shell is grown around the quantum dot with a very small taper towards the tip $\left(\sim 1^{\circ}\right)$ to boost single-photon collection efficiency by guiding the light efficiently towards the collection optics [18]. Second, by design, the single quantum dot is on the nanowire waveguide axis, which is needed for efficient coupling of the dipole emitter to the fundamental waveguide mode [19]. Finally, the nanowire consists of a 
(a)

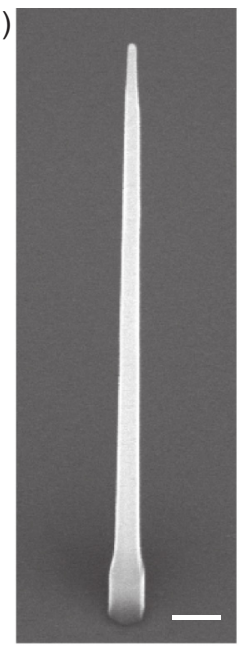

(b)

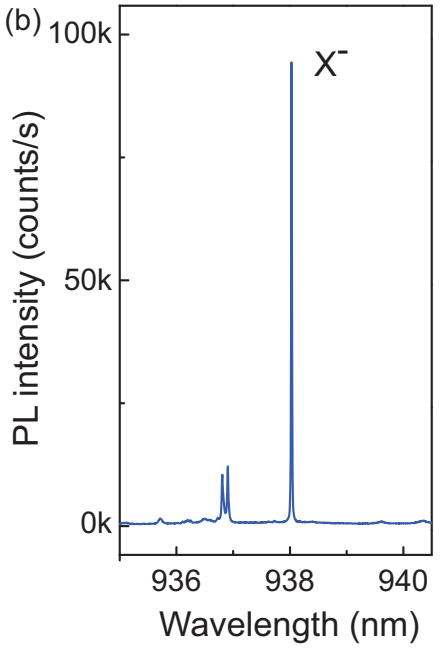

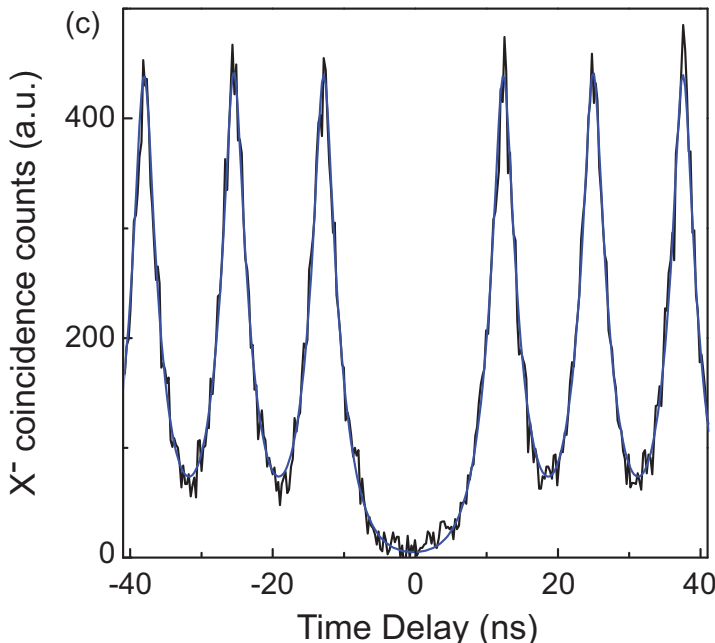

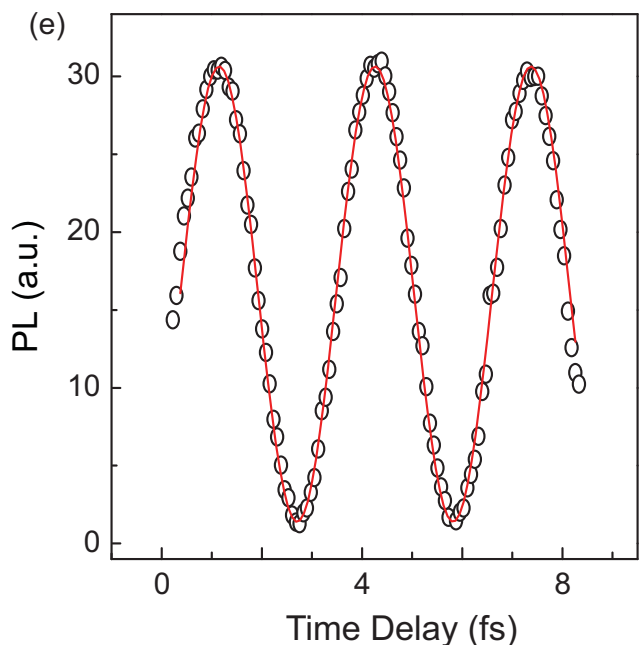

Time Delay (fs)

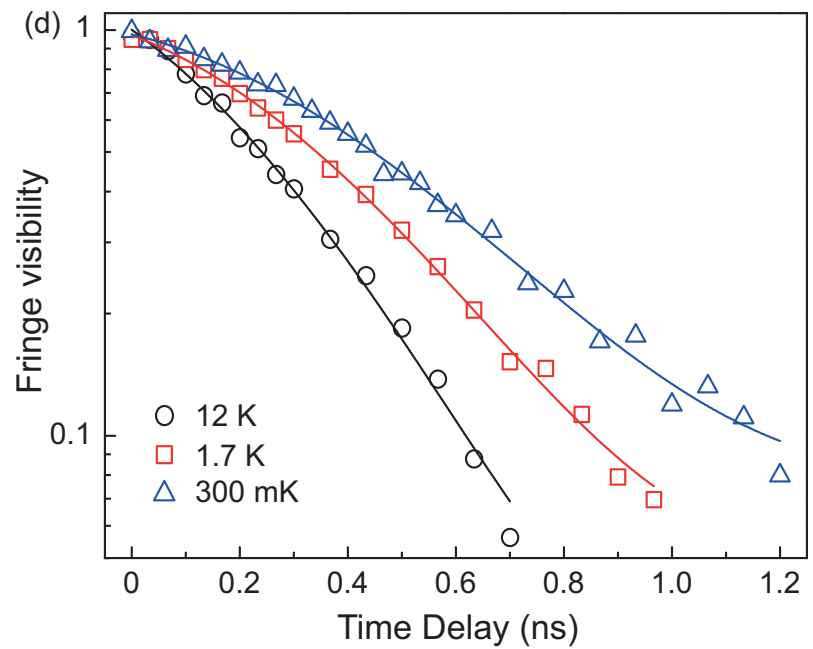

FIG. 1. Single-photon interference measurements. (a) SEM image of tapered nanowire waveguide containing a single quantum dot. Scale bar: $200 \mathrm{~nm}$. (b) Typical PL spectrum of a quantum dot that is predominantly charged. $\mathrm{X}^{-}$: charged exciton. (c) Autocorrelation measurement of $\mathrm{X}^{-}$demonstrating a single-photon purity of greater than $99 \%$. A fit to the data (blue line) yields $g^{2}(0)<0.008$. (d) Single-photon interference measurements of a single quantum dot at $300 \mathrm{mK}$ (blue triangles), $1.7 \mathrm{~K}$ (red squares), and $12 \mathrm{~K}$ (black circles). The solid lines are a fit to the data using a Voigt profile (described in the text). (e) Raw single-photon interference fringes with a step size of $20 \mathrm{~nm}$ at maximum fringe visibility for a temperature of $300 \mathrm{mK}$.

pure wurtzite InP crystal structure that is free of stacking faults. Importantly, this crystal phase purity of the nanowire waveguide is obtained for core diameters of $25 \mathrm{~nm}$ or less and is maintained during shell growth to construct the waveguide [20]. The crystal phase purity is essential in obtaining a narrow emission spectrum: the measured linewidths are reduced by more than two orders of magnitude as compared to when stacking faults were present in nanowires [18,21-23]. Typically, stacking faults in the nanowire act as efficient charge traps, which is detrimental to the quantum dot emission linewidth owed to spectral wandering.

As an added advantage, the nanowire heterostructures presented in this work are also deterministically positioned by combining both electron beam patterning and selective area epitaxy [20,23]. The main important features pertaining to the single-photon quality that we demonstrate are long coherence, high light collection efficiency, and suppressed multiphoton emission. These main attributes when combined together have not been achieved to date for site-controlled quantum dots [19,22,24-27].

\section{SINGLE-PHOTON COHERENCE MEASUREMENTS}

To determine the coherence length of single photons originating from quantum dots in nanowire waveguides and obtain a high resolution measurement of the emission spectrum, we employ field-correlation measurements using a Michelson interferometer [28-30]. To ensure good overlap of the spatial modes between both paths, the quantum dot emission is coupled to a single-mode fiber before entering the Michelson interferometer. When the path difference between both paths is varied we observe single-photon interference through oscillations in the measured intensity at the output, where the decay of the interference fringe visibility is set by the temporal coherence of the source. 

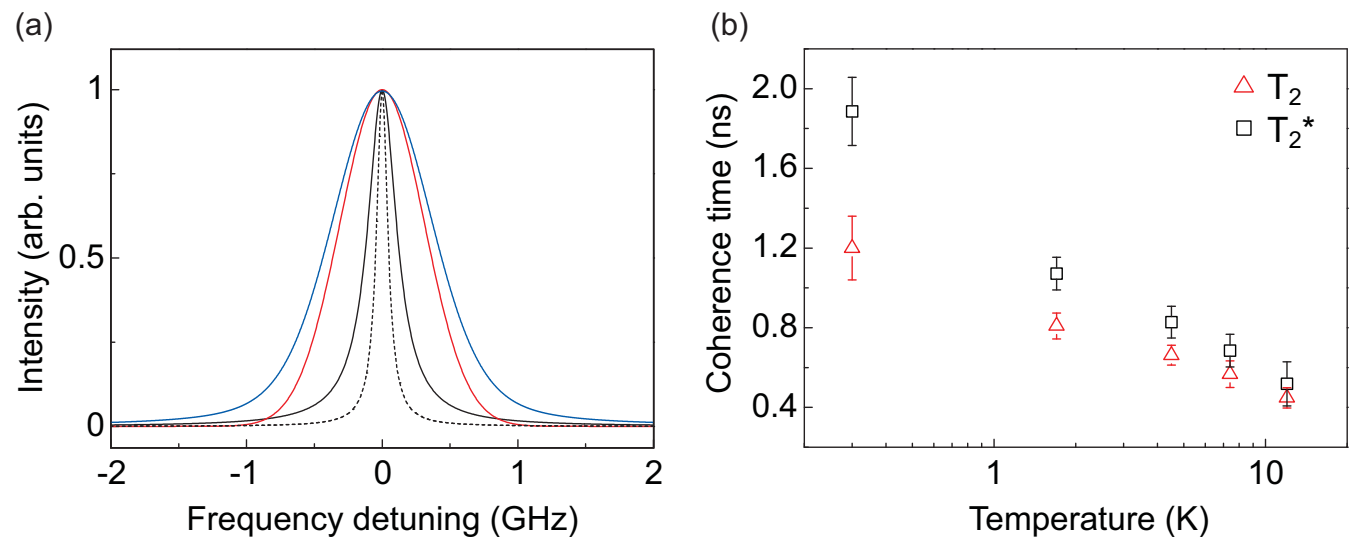

FIG. 2. Single quantum dot coherence. (a) Single quantum dot emission linewidth is represented by a Voigt profile (blue) with a full width at half maximum of $880(130) \mathrm{MHz}$, which is a convolution of a Lorentzian (black) and Gaussian (red) line shape. The parameters for the homogeneous broadening [Lorentzian, $T_{2}=1.2(2) \mathrm{ns}$, corresponding to $260(40) \mathrm{MHz}$ ] and inhomogeneous broadening [Gaussian, $T_{c}=0.9(1) \mathrm{ns}$, corresponding to $\left.730(50) \mathrm{MHz}\right]$ are extracted from the fit of the Michelson data at $300 \mathrm{mK}$ in Fig. 1(d). For comparison, the dotted black line shows the lifetime Fourier-transform limit of $100 \mathrm{MHz}$. (b) Coherence time, $T_{2}$, and corresponding pure dephasing time, $T_{2}^{*}$, extracted from the fits of Fig. 1(d). At $300 \mathrm{mK}$ the pure dephasing time is longer than the exciton lifetime, $T_{1}$, of 1.6(1) ns.

The photoluminescence spectrum of the quantum dot used for the field-correlation measurements is presented in Fig. 1(b). The brightest line for this particular quantum dot at $938 \mathrm{~nm}$ is the singly charged exciton, $\mathrm{X}^{-}$. Assignment of $\mathrm{X}^{-}$is confirmed by a combination of both polarization analysis as in Ref. [20] and cross-correlation measurements (see Supplemental Material [31], Figs. S3 and S4). At saturation of the quantum dot emission, we measured count rates for $\mathrm{X}^{-}$of 460 kilocounts per second on a silicon avalanche photodiode under pulsed excitation. Importantly, this high measured count rate is achieved while maintaining the single-photon purity of greater than $99 \%$ for the source; see Fig. 1(c). We excite the dot above the nanowire band gap at $750 \mathrm{~nm}$ using 3 ps laser pulses at a repetition rate of $76 \mathrm{MHz}$. Accounting for a transmission of $1.4(1) \%$ in the optical setup, we calculate a single-photon source efficiency for $\mathrm{X}^{-}$of 43(4)\%. The single-photon source efficiency can be further enhanced by integrating a bottom gold mirror with a thin dielectric at the base in order to collect the downward emitted photons that are lost [18,32].

The results of the single-photon interference measurements are presented in Fig. 1(d) for three different temperatures from $12 \mathrm{~K}$ down to $300 \mathrm{mK}$. Figure 1(e) depicts the raw interference fringes of the charged exciton line at $300 \mathrm{mK}$ obtained around zero path difference between both arms of the Michelson interferometer for a step size of $20 \mathrm{~nm}$. A fit to the data (red line) yields a maximum raw fringe visibility of $95 \%$. Correcting for the Michelson system response, a maximum fringe visibility of $99 \%$ is attained (see Methods). For increasing path difference (time delay), a decay in the fringe visibility is observed and we extract the coherence length and corresponding coherence time of the emitted single photons.

We now discuss the nature of the line shape that is obtained from the single-photon interference measurements in Fig. 1(d). We observe a Voigt profile, which is a Gaussian convolved with a Lorentzian. Gaussian line broadening is due to charge fluctuations in the quantum dot environment, leading to spectral wandering on time scales longer than the exciton lifetime, but shorter than the measurement time needed to acquire a visibility data point $(\sim 5$ minutes in our measurements). From a fit to the data at $300 \mathrm{mK}$ in Fig. 1(d) (fit: blue line; data: open triangles), we extract a homogeneous (Lorentzian) linewidth of 260(40) $\mathrm{MHz}$ and inhomogeneous (Gaussian) linewidth of $730(50) \mathrm{MHz}$ (see Methods). Both components of the Voigt profile are plotted in Fig. 2(a), represented by the black (Lorentzian) and red (Gaussian) line. The convolution of both line shapes yields the measured Voigt profile with a full width at half maximum of $880(130) \mathrm{MHz}$, shown in blue. For comparison the dotted black line shows the lifetime Fourier-transform limit of $100 \mathrm{MHz}$, for our measured exciton lifetime, $T_{1}$, of 1.6(1) ns (see Supplemental Material [31], Fig. S2: dot A). The deviation from the Fourier-transform limit is mainly due to Gaussian line broadening. We point out that this Gaussian line broadening mechanism due to charge fluctuations in the environment is also observed in integrated resonant fluorescence [8,9], and found to degrade the visibility of two-photon interference from independent quantum dots [33-35].

\section{COUNTERACTING DEPHASING MECHANISMS}

In Fig. 2(b), we present the homogeneous coherence time, $T_{2}$, as extracted from the Voigt profile and corresponding pure dephasing time, $T_{2}^{*}$, from the relation

$$
\frac{1}{T_{2}}=\frac{1}{2 T_{1}}+\frac{1}{T_{2}^{*}},
$$

as a function of temperature. Here, $T_{2}^{*}$ is the pure dephasing, and $T_{2}$ is the homogeneous coherence time that includes both relaxation and pure dephasing processes. Decreasing the temperature results in an enhancement of the singlephoton coherence as interactions with phonons are suppressed. Both $T_{2}$ and $T_{2}^{*}$ show an exponential increase with cooling. Interestingly, the homogeneous coherence time doubles to $1.2(2) \mathrm{ns}$ when cooling from $4.5 \mathrm{~K}$ to $300 \mathrm{mK}$, while the pure dephasing time surpasses the measured exciton lifetime at $300 \mathrm{mK}$. The corresponding inhomogeneously broadened linewidth reduces at a much weaker rate from $1.42 \mathrm{GHz}$ at 

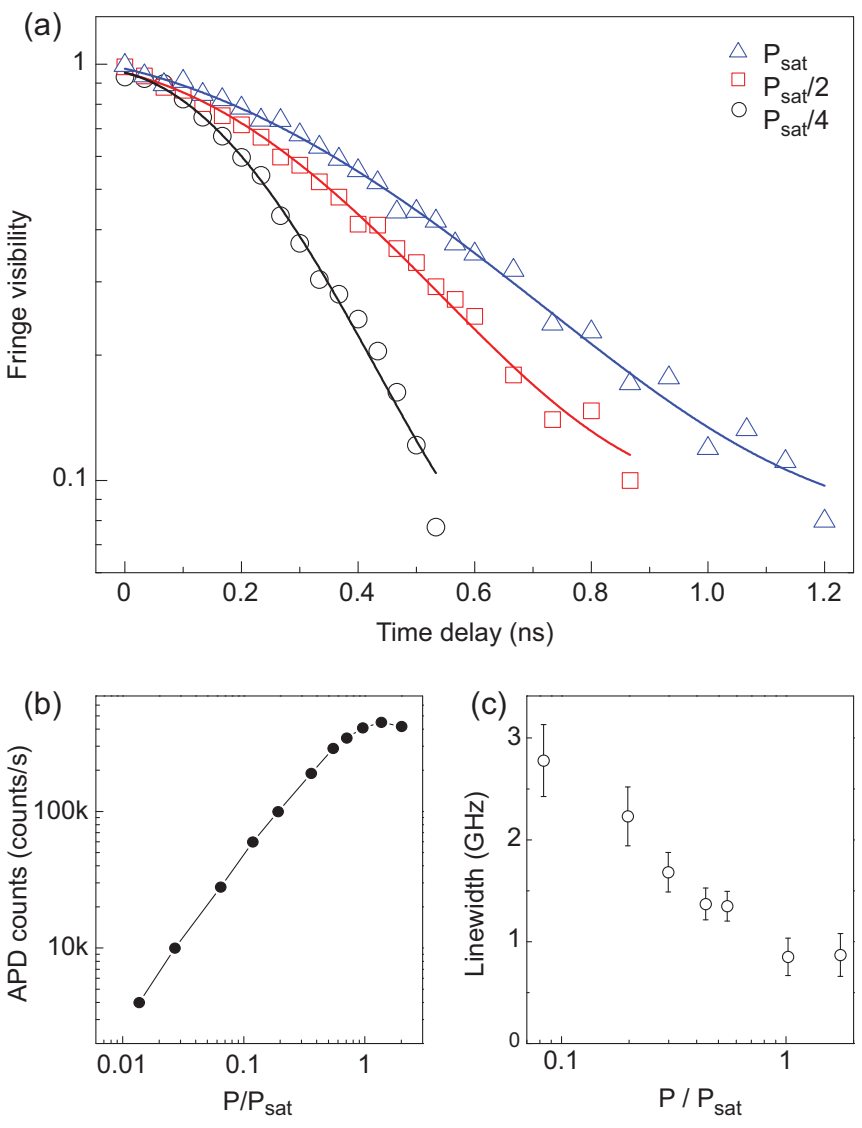

FIG. 3. Power narrowing of the single quantum dot emission linewidth. (a) Single-photon interference measurements of a single quantum dot at $300 \mathrm{mK}$ as a function of excitation power, $P$. In contrast to conventional self-assembled quantum dots, importantly, the best coherence is observed at exciton saturation of the quantum dot emission where the detected count rate is highest. The laser power, $P_{\text {sat }}$, used to saturate the exciton transition is $\sim 3 \mu \mathrm{W}$ (spot size of $\sim 1 \mu \mathrm{m}$ ). (b) Power dependence of charged exciton emission line. The exciton emission saturates at 460000 counts per second as measured on a single-photon avalanche photodiode. (c) Emission linewidth as a function of excitation power, $P$, as extracted from a fit to the data by a Voigt profile as presented in (a). $P_{\text {sat }}$ corresponds to the excitation power used to saturate the charged exciton transition.

$12 \mathrm{~K}$ to $880 \mathrm{MHz}$ at $300 \mathrm{mK}$ (see Supplemental Material [31], Fig. S5). The observed reduction in emission linewidth with temperature is consistent with previous work where quantum dot emission linewidths were reduced by as much as $120 \mathrm{MHz} / \mathrm{K}[36,37]$.

Finally, we present in Fig. 3(a) the single-photon interference measurements at $300 \mathrm{mK}$ as a function of excitation power. Remarkably, we observe the longest coherence at saturation of the quantum dot emission where the measured count rates on the single-photon detector are highest [see Fig. 3(b)]. The resulting emission linewidth, to be discussed subsequently, is reduced from $2.7(3) \mathrm{GHz}$ at the lowest excitation power used to $0.8(2) \mathrm{GHz}$ at saturation of the quantum dot emission [see Fig. 3(c)]. Excellent fits to the data in Fig. 3(a) are made by fixing $T_{2}$ to $1.2(2) \mathrm{ns}$ at the saturation power, $P_{\text {sat }}$, of the quantum dot emission and increasing the degree of Gaussian line broadening as the excitation power is reduced. The fact that $T_{2}$ is power independent follows from the data. Homogeneous broadening, affecting $T_{2}$, can be caused by carrier-phonon and carrier-carrier interactions that take place within the exciton lifetime. Carrier-phonon interactions depend on temperature; as is clearly observed in Fig. 2(b), the homogeneous coherence time increases with reduced temperature. In contrast, carrier-carrier interactions depend on the power. But since the carriers are decaying quickly or trapped within picosecond time scales as compared to the exciton lifetime of $1.6 \mathrm{~ns}$, the exciton is free from homogeneous broadening through carrier-carrier interactions for $99.9 \%$ of its lifetime.

We point out that the observation of power narrowing for the single quantum dot emission spectrum is not limited to $300 \mathrm{mK}$, but is also observed at temperatures up to $12 \mathrm{~K}$ (see Supplemental Material [31], Fig. S6). This outcome suggests that the activation energy of charge traps in the nanowire is above $12 \mathrm{~K}$, consistent with photoluminescence measurements in our previous work [20]. In all of our samples, we observe weak emission associated with donor-acceptor levels, at approximately $50 \mathrm{meV}$ below the pure wurtzite InP nanowire peak at $1.49 \mathrm{eV}$ [20].

\section{PHOTON INDISTINGUISHABILITY}

In the following, we analyze the degree of photon indistinguishability at a temperature of $300 \mathrm{mK}$ and for two excitation powers: at saturation of the quantum dot emission where the single photons exhibit the longest coherence and five times below saturation. We perform Hong-Ou-Mandel (HOM) two-photon interference experiments of two subsequently created photons from a similar nanowire quantum dot (dot B in the Supplemental Material [31], Fig. S2). The HOM effect predicts that two perfectly indistinguishable photons, thus Fourier-limited photons, entering a nonpolarizing beam splitter will coalesce in only one of the two exit ports. The HOM experimental setup is depicted in Fig. 4(a), adapted from Santori et al. [38]. Here, the nanowire, cooled to 300 $\mathrm{mK}$, is excited with a pulsed picosecond supercontinuum laser ( $\sim 6 \mathrm{ps}$ ) operating at $750 \mathrm{~nm}$ and a repetition rate of $20 \mathrm{MHz}$. A $\lambda / 2$ half-wave plate is used to render the two photons distinguishable or indistinguishable.

Figures 4(b) and 4(c) show the HOM two-photon interference for an excitation power at saturation $\left(P_{\text {sat }}\right)$ and for one-fifth of the saturation power $\left(P_{\text {sat }} / 5\right)$, respectively. With the use of fast superconducting nanowire detectors $(\sim 40 \mathrm{ps}$ time resolution) as compared to the homogeneous coherence time, the HOM dip at zero time delay can be accurately resolved even in the case of single photons not at the Fouriertransform limit $[39,40]$. Here, the width of the dip depends on both the emission time jitter of the single-photon source and inhomogeneous broadening due to charge fluctuations in the environment. From a fit to the HOM two-photon interference data at saturation using an extended theory of Legero et al. [39] (see Supplemental Material [31]), we extract a 1/e width of the HOM dip of $0.83(2) \mathrm{ns}$ and inhomogeneous broadening of 1.17(3) GHz. Below saturation (five times lower power), a $1 / e$ width of the HOM dip of $0.81(2) \mathrm{ns}$ and inhomogeneous broadening of 1.21(4) GHz is attained. The fact that we observe an inhomogeneous broadening in the HOM measurements 

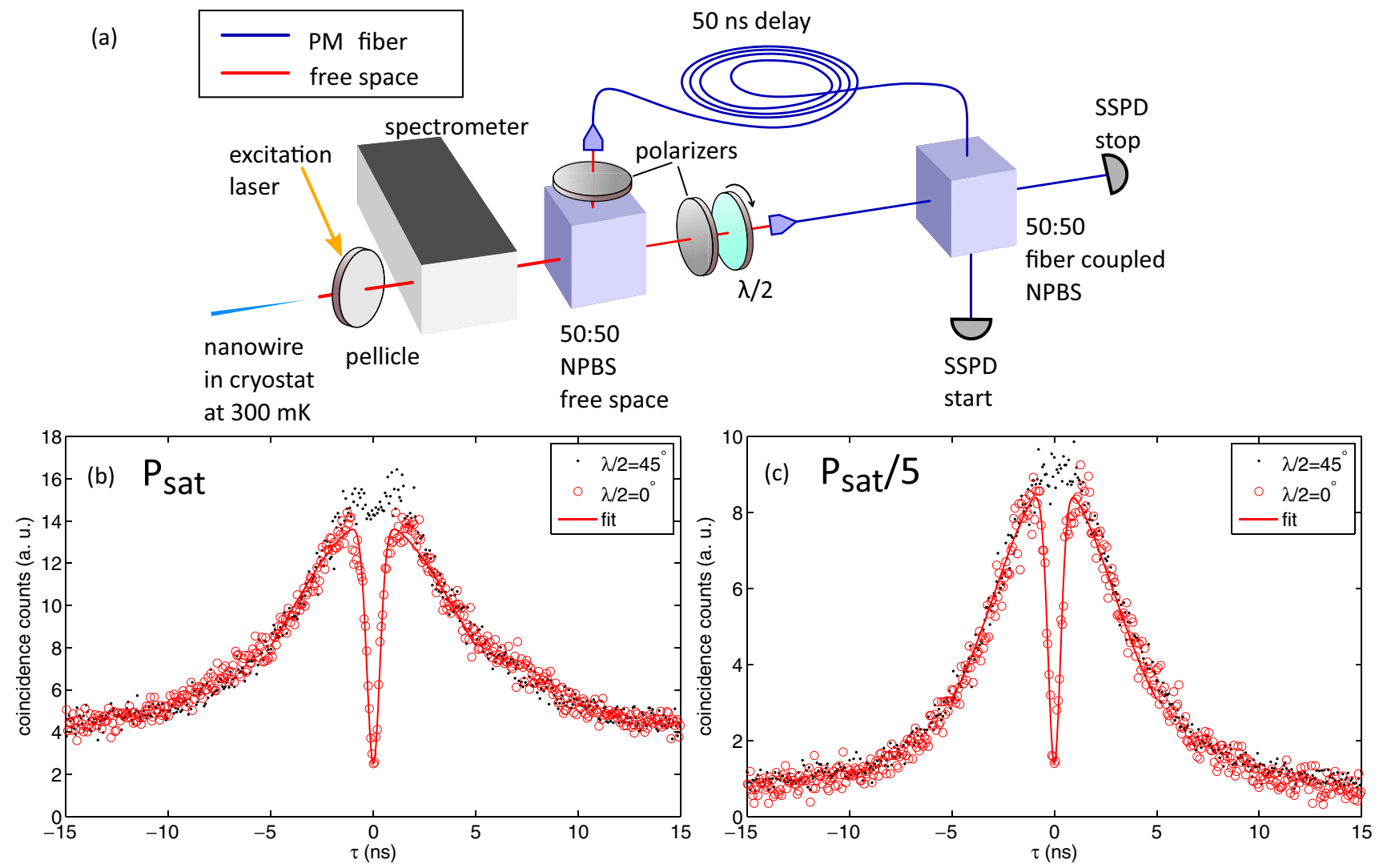

FIG. 4. Photon indistinguishability. (a) Schematic of HOM setup for two subsequently created photons from a quantum dot in a nanowire. The charged exciton line of the quantum dot emission is filtered in a spectrometer. A nonpolarizing beam splitter (NPBS) splits the photons in a short and long path. The delay is precisely matched to the repetition rate of the laser $(20 \mathrm{MHz})$ so that the two photons interfere at the fiber-coupled NPBS. That is, a single photon traveling the long path interferes with the next subsequent photon traveling the short path all in polarization-maintaining (PM) fibers. SSPD: Superconducting nanowire single photon detector. (b) HOM interference measured at saturation for parallel (indistinguishable case) and perpendicular polarizations (distinguishable case). (c) HOM interference as in (b), but for one-fifth of the saturation power. The coincidence data in (b) and (c) consist of 64 ps time bins.

comparable to the single-photon coherence measurements suggests that our measurements are still susceptible to noise above $20 \mathrm{MHz}$. This observation is in stark contrast to two-photon interference measurements of subsequent photons created by InGaAs quantum dots [9], as the spectral noise of the electric and magnetic field falls off rapidly above $50 \mathrm{kHz}$ [41]. One physical mechanism that could lead to noise above $20 \mathrm{MHz}$ in our case is fast cotunneling processes of charges from the nanowire surface [42]. These fast tunneling processes could be suppressed, in principle, by increasing the nanowire diameter or by passivating the nanowire surface.

The visibility of the HOM interference is presented in Table I. When integrated over the whole central peak the visibility is relatively low, less than $10 \%$, due to inhomogeneous broadening and the nonresonant excitation process leading to an emission time jitter. However, in conjunction with the long coherence length of the emitted photons, high collection efficiency, and fast superconducting nanowire detectors, it is possible to reach a high visibility for two-photon interference by time gating. For the narrowest time window employed we reach raw $\mathrm{HOM}$ visibilities as high as $83 \% \pm 3 \%$, but at a cost of photon count rates (see Table I). Still, the two-photon visibility could be improved by resonant excitation to reduce the inhomogeneous broadening and emission time jitter $[43,44]$ while using a weak laser to fill the traps [10]. We also expect that the two-photon interference visibility would significantly increase if the temporal separation between two consecutively emitted photons by the same quantum dot is reduced as shown in recent work by Thoma et al. [45]. An electric field could also be potentially used to increase the two-photon visibility by emptying charge traps in the vicinity of the quantum dot as demonstrated recently in an electrically controlled cavity structure [44].

TABLE I. Raw HOM visibilities for different integration widths, not corrected for setup imperfections such as an imbalanced beam splitter ratio, and corresponding counts as a percentage of the maximum brightness. Note: 30 ns corresponds to full peak integration.

\begin{tabular}{llcccc}
\hline \hline & $30 \mathrm{~ns}$ & $1 \mathrm{~ns}$ & $0.5 \mathrm{~ns}$ & $64 \mathrm{ps}$ \\
\hline $\boldsymbol{P}_{\text {sat }}$ & HOM visibility (\%) & 4.5 & $60 \pm 4$ & $76 \pm 4$ & $83 \pm 3$ \\
& Counts (\%) & 100 & 44.4 & 25 & 3.6 \\
$\boldsymbol{P}_{\text {sat }} / \mathbf{5}$ & HOM visibility (\%) & 8.4 & $61 \pm 3$ & $73 \pm 3$ & $85 \pm 3$ \\
& Counts (\%) & 20 & 8.9 & 5 & 0.7 \\
\hline \hline
\end{tabular}


(a)
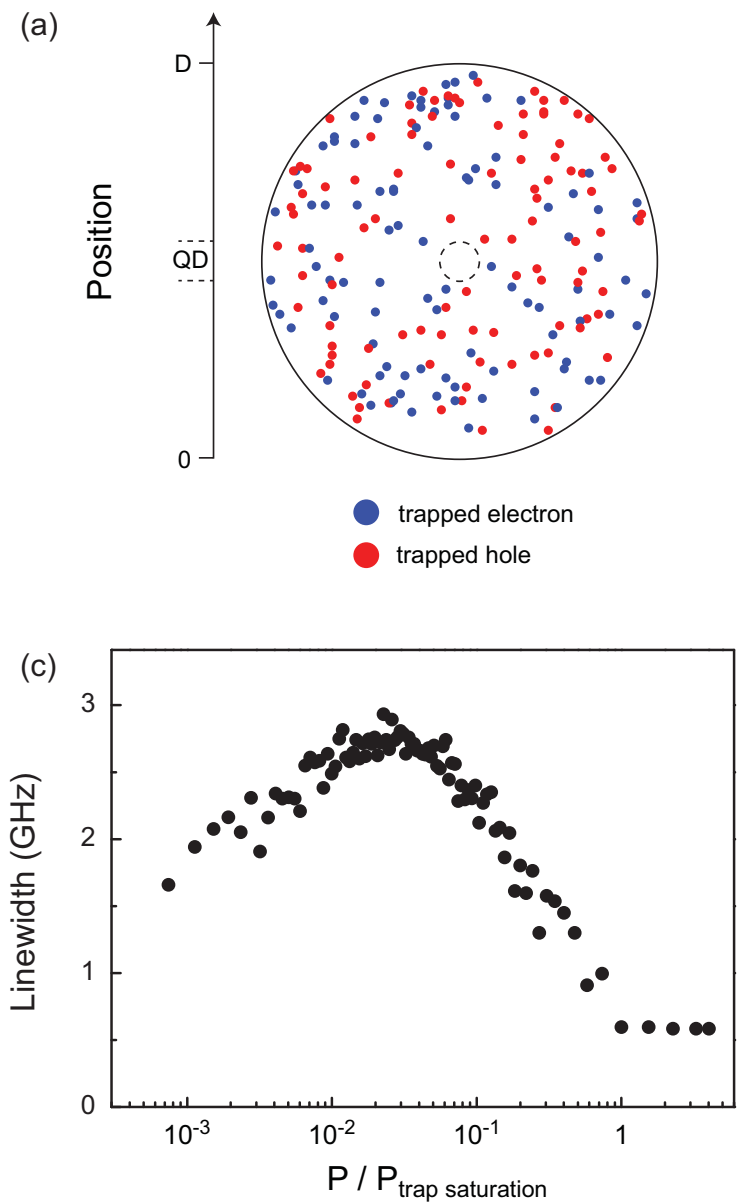
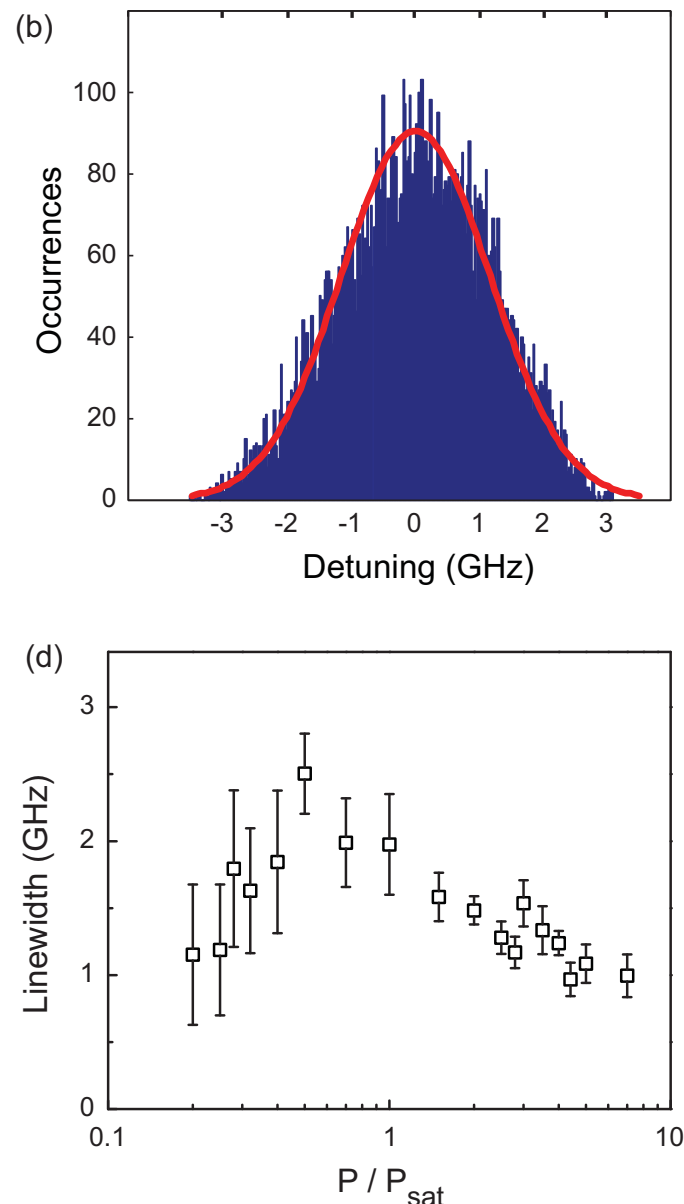

FIG. 5. Monte Carlo simulations of Gaussian line broadening mechanism for single quantum dot emission line. (a) A projection of randomly positioned trap sites for both electrons (blue) and holes (red dots) over a $400 \mathrm{~nm}$ nanowire segment. The nanowire diameter, $D$, is $200 \mathrm{~nm}$. The trap density used for the simulations to reproduce the measured exciton linewidth as a function of power is $\sim 1 \times 10^{16} \mathrm{~cm}^{-3}$. The quantum dot, QD, in the center of the nanowire is represented as a dotted circle with diameter of $20 \mathrm{~nm}$. (b) Resulting histogram of Stark shift induced on the exciton transition for 10000 possible charge configurations distributed across trap sites surrounding the quantum dot for an average trap occupancy probability of 0.5 . Due to the random nature of the possible charge configurations, the broadening mechanism of the emission line is a Gaussian (fit curve in red). (c) Monte Carlo simulations showing the quantum dot emission linewidth as a function of excitation power. The simulations are in excellent agreement with the experimental power dependent emission linewidth of dot B presented in (d). $P_{\text {trap saturation }}$ corresponds to the laser power used to fill trap sites, whereas $P_{\text {sat }}$ corresponds to the excitation power used to saturate the charged exciton transition.

\section{MONTE CARLO SIMULATIONS OF THE QUANTUM DOT EMISSION SPECTRUM}

We now present Monte Carlo simulations of the quantum dot emission spectrum, which take into account charge fluctuations in the quantum dot environment as a function of power. To reproduce the exciton emission linewidth, we model 1000 randomly positioned electron and hole traps in the nanowire, corresponding to a trap density of $\sim 1 \times 10^{16} \mathrm{~cm}^{-3}$. This trap density gives approximately 50 trap sites within a radius of $200 \mathrm{~nm}$ from the dot. Figure 5(a) displays the random positioning of electron (blue) and hole (red) trap sites in the nanowire. In our experiments, the excitation process above the nanowire band gap excites both the quantum dot and carriers in the nanowire, which subsequently fill trap sites. The interaction between the trapped charges and the quantum dot exciton results in a shift of the exciton emission energy via the Stark effect. The result of the Monte Carlo simulations iterating over 10000 possible charge configurations distributed across the randomly positioned trap sites for a trap occupation probability of 0.5 is shown in Fig. 5(b). Owing to the random nature of the charge fluctuations, the resulting broadening of the emission line is a Gaussian (red fit to the histogram).

Our Monte Carlo simulation gives the emission linewidth as a function of trap occupancy. The correspondence between laser power and trap occupancy follows a nonlinear relation and is calculated with a rate equation model (see Supplemental Material [31]). The simulation results of the emission linewidth as a function of excitation power are shown in Fig. 5(c). First, we observe that the linewidth broadens with increasing excitation power. This is the typical power broadening regime as is also observed for self-assembled quantum dots. Next, the broadest emission linewidth is attained, corresponding to the situation where half of the trap sites are occupied, thereby providing the highest number of possible charge configurations in the quantum dot environment. Here, the system reaches the 
highest entropy. By further increasing the excitation power, the power-narrowing regime is reached corresponding to a decrease in the emission linewidth of $2.5 \mathrm{GHz} /$ decade. In this power-narrowing regime, the higher number of trap sites that are occupied results in a reduction of the disorder in the quantum dot environment. The linewidth of the dot may therefore be used to probe the entropy of the system. Finally, once nearly all of the charge traps are occupied at higher power no further improvement of the emission linewidth is expected. The simulation results are in excellent agreement with the observed emission linewidth of dot B for increasing excitation power as presented in Fig. 5(d). We observe both the power broadening (for low powers) and narrowing regimes (for higher powers), including the decrease in emission linewidth of $1.9 \mathrm{GHz}$ per decade for increasing power and eventual saturation of this reduction.

It is worth mentioning why we reach the power-narrowing regime for the first time. We attribute this to a unique combination of both the nanowire geometry and reduced trap density in ultraclean wurtzite InP nanowires. Because of these two features photogenerated charges in the nanowire remain localized around the quantum dot and may be used to saturate nearly all of the surrounding charge traps. In contrast, in a bulk sample, saturating surrounding trap sites is extremely difficult owing to a significant increase in available material. Thus, for a bulk sample, carriers may diffuse to trap sites located farther away from the quantum dot. This outcome leaves self-assembled quantum dots in the power-broadening regime. However, careful design of the photonic structure in order to reduce the surrounding material, such as etching photonic nanowires similarly to the work of Claudon et al. [32], may enable the power-narrowing regime to be reached for self-assembled quantum dots. On the other hand, recent work with electrically controlled quantum dot-cavity structures have shown it is possible to empty the charge traps in the vicinity of the quantum dot in order to significantly improve the photon indistinguishability [44].

\section{CONCLUSION}

We have shown single-photon coherence measurements from a quantum dot in a nanowire waveguide and found a homogeneous coherence time of $1.2 \mathrm{~ns}$ at $300 \mathrm{mK}$. Importantly, the longest single-photon coherence that we observe is not attained at very low excitation powers, but at the excitation power where the quantum dot single-photon emission rate is the highest. We studied the degree of photon indistinguishability for this case of enhanced coherence via two-photon HOM interference measurements of subsequently created photons from the same quantum dot. The two-photon interference revealed a two-photon visibility as high as $83 \%$ under strict temporal postselection. The observed visibility of two-photon interference may be improved in future work by reducing the inhomogeneous broadening and emission timing jitter of the source by resonant excitation, reducing the temporal separation between subsequent photons, and by applying an electric field to reduce charge noise.

We overcame power broadening by growing ultraclean wurtzite InP nanowires without stacking faults to reduce the trap density. This reduced trap density combined with the nanowire geometry allowed us to fill nearly all of the trap sites surrounding the quantum dot at higher excitation powers, resulting in a narrower emission spectrum. Finally, the enhanced single-photon coherence, brightness, and purity that we demonstrate brings this young field of quantum dots in nanowires among the leading candidates for implementations of single-photon sources in future quantum and photonic technologies.

\section{METHODS}

\section{A. Single-photon interference fringe visibility}

The interference fringe visibility is defined as

$$
V=\frac{I_{\max }-I_{\min }}{I_{\max }+I_{\min }},
$$

where $I_{\max }$ and $I_{\min }$ correspond to the case of optimal constructive and destructive interference, respectively. We measure a maximum raw interference fringe visibility for the quantum dot of $95 \%$, whereas for a coherent laser with 4 $\mathrm{MHz}$ linewidth we obtain a raw interference fringe visibility of $96 \%$. In all of the single-photon interference measurements, we present the fringe visibility corrected for the Michelson system response (see Supplemental Material [31], Fig. S1). This correction is needed due to the limited phase stability of the Michelson setup caused by vibrations and small temperature fluctuations.

\section{B. Relationship between coherence and linewidth}

The form of the fringe visibility decay, $g^{(1)}(\tau)$, for coherent light depends on the type of spectral broadening that applies. For light with a Lorentzian line shape, the decay of the fringe visibility is exponential, where

$$
g^{(1)}(\tau) \sim \exp \left(-\frac{|\tau|}{T_{2}}\right) .
$$

The relationship between the full width at half maximum in frequency, $\Delta f$, and the coherence time, $T_{2}$, for the Lorentzian line shape is given by

$$
\Delta f_{L}=\frac{1}{\pi T_{2}}
$$

whereas for a Gaussian line shape, the fringe visibility decay follows the form

$$
g^{(1)}(\tau) \sim \exp \left[-\frac{\pi}{2}\left(\frac{\tau}{\tau_{c}}\right)^{2}\right] .
$$

In this case, the full width at half maximum for a Gaussian is

$$
\Delta f_{G} \sim \frac{\sqrt{2 \ln 2}}{\sqrt{\pi} \tau_{c}},
$$

where $\tau_{c}$ is the coherence time for the Gaussian component. The decay of the fringe visibility was fitted for all data using a Voigt profile. In the time domain the Voigt profile is a product of a Gaussian and Lorentzian:

$$
g^{(1)}(\tau) \sim \exp \left[-\frac{\pi}{2}\left(\frac{\tau}{\tau_{c}}\right)^{2}-\frac{|\tau|}{T_{2}}\right] .
$$


In the frequency domain the full width at half maximum of the resulting Voigt profile is [35]

$$
\Delta f_{V}=0.535 \Delta f_{L}+\sqrt{0.217 \Delta f_{L}^{2}+\Delta f_{G}^{2}} .
$$

\section{ACKNOWLEDGMENTS}

We acknowledge Klaus Jöns for technical support and scientific discussions, as well as Sander Dorenbos, Sergiy Dobrovolskiy, and Niels Los for the Single Quantum EOS 210 closed-cycle system used in this work consisting of fast superconducting nanowire single photon detectors with high time resolution $(\sim 40 \mathrm{ps})$. This work was supported by the European Union Seventh Framework Programme 209 (FP7/2007-2013) under Grant Agreement No. 601126210 (HANAS), the Dutch Organization for Fundamental Research on Matter (FOM), and Industry Canada. M.E.R., R.W.H., and
V.Z. designed and conceived the project. M.E.R. and G.B. performed the experiments with R.W.H. providing software expertise for the single-photon interference measurements. A.F. and A.R. performed the HOM experiments at $300 \mathrm{mK}$ with input from M.E.R. and V.Z. T.B. and M.E.R. performed the cross-correlation measurements, presented in the Supplemental Material [31], that were carried out in the laboratory of S.H. and M.K. in Würzburg. M.E.R. analyzed the data with input from G.B., A.F., B.J.W., M.A.M.V., and V.Z. G.B. performed the Monte Carlo simulations with input from M.E.R., B.J.W., M.A.M.V., and V.Z. D.D. and P.J.P. carried out the nanowire quantum dot growth. D.D. and J.L. performed the processing required prior to the site-selected nanowire quantum dot growth. M.E.R. led the project under supervision of V.Z. M.E.R. wrote the paper with input from all authors. All authors discussed the results and commented on the manuscript.

M.E.R., G.B., and A.F. contributed equally to this work.
[1] H. J. Kimble, The quantum internet, Nature (London) 453, 1023 (2008).

[2] E. Knill, R. Laflamme, and G. J. Milburn, A scheme for efficient quantum computation with linear optics, Nature (London) 409 , 46 (2001).

[3] D. Fattal, E. Diamanti, K. Inoue, and Y. Yamamoto, Quantum Teleportation with a Quantum Dot Single Photon Source, Phys. Rev. Lett. 92, 037904 (2004).

[4] J. Hofmann, M. Krug, N. Ortegel, L. Gérard, M. Weber, W. Rosenfeld et al., Heralded entanglement between widely separated atoms, Science 337, 72 (2012).

[5] H. Bernien, B. Hensen, W. Pfaff, G. Koolstra, M. S. Blok, L. Robledo et al., Heralded entanglement between solid-state qubits separated by three meters, Nature (London) 497, 86 (2013).

[6] H.-J. Briegel, W. Dür, J. I. Cirac, and P. Zoller, Quantum Repeaters: The Role of Imperfect Local Operations in Quantum Communication, Phys. Rev. Lett. 81, 5932 (1998).

[7] A. Berthelot, I. Favero, G. Cassabois, C. Voisin, C. Delalande, $\mathrm{Ph}$. Roussigno et al., Unconventional motional narrowing in the optical spectrum of a semiconductor quantum dot, Nat. Phys. 2, 759 (2006).

[8] S. Ates, S. M. Ulrich, S. Reitzenstein, A. Löffler, A. Forchel, and P. Michler, Postselected Indistinguishable Photons from the Resonance Fluorescence of a Single Quantum Dot in a Microcavity, Phys. Rev. Lett. 103, 167402 (2009).

[9] Y.-M. He, Y. He, Y.-J Wei, D. Wu, M. Atatüre, and C. Schneider, On-demand semiconductor single-photon source with nearunity indistinguishability, Nat. Nanotechnol. 8, 213 (2013).

[10] O. Gazzano, S. Michaelis de Vasconcellos, C. Arnold, A. Nowak, E. Galopin, I. Sagnes et al., Bright solid-state sources of indistinguishable single photons, Nat. Commun. 4, 1425 (2013).

[11] H. D. Robinson and B. B. Goldberg, Light-induced spectral diffusion in single self-assembled quantum dots, Phys. Rev. B 61, R5086(R) (2000).

[12] S. Stufler, P. Ester, A. Zrenner, and M. Bichler, Power broadening of the exciton linewidth in a single InGaAs/GaAs quantum dot, Appl. Phys. Lett. 85, 4202 (2004).
[13] M. E. Ware, E. A. Stinaff, D. Gammon, M. F. Doty, A. S. Bracker, D. Gershoni, V. L. Korenev, S. C. Badescu, Y. Lyanda-Geller, and T. L. Reinecke, Polarized Fine Structure in the Photoluminescence Excitation Spectrum of a Negatively Charged Quantum Dot, Phys. Rev. Lett. 95, 177403 (2005).

[14] A. Muller, E. B. Flagg, P. Bianucci, X. Y. Wang, D. G. Deppe, W. Ma, J. Zhang, G. J. Salamo, M. Xiao, and C. K. Shih, Resonance Fluorescence from a Coherently Driven Semiconductor Quantum Dot in a Cavity, Phys. Rev. Lett. 99, 187402 (2007).

[15] J. Houel, A. V. Kuhlmann, L. Greuter, F. Xue, M. Poggio, B. D. Gerardot et al., Probing Single-Charge Fluctuations at a GaAs/AlAs Interface Using Laser Spectroscopy on a Nearby InGaAs Quantum Dot, Phys. Rev. Lett. 108, 107401 (2012).

[16] J. Suffczynski, A. Dousse, K. Gauthron, A. Lemaître, I. Sagnes, L. Lanco, J. Bloch, P. Voisin, and P. Senellart, Origin of the Optical Emission within the Cavity Mode of Coupled Quantum Dot-Cavity Systems, Phys. Rev. Lett. 103, 027401 (2009).

[17] M. Winger, T. Volz, G. Tarel, S. Portolan, A. Badolato, K. J. Hennessy et al., Explanation of Photon Correlation in the FarOff-Resonance Optical Emission from a Quantum Dot-Cavity System, Phys. Rev. Lett. 103, 207403 (2009).

[18] M. E. Reimer, G. Bulgarini, N. Akopian, M. Hocevar, M. B. Bavinck, M. A. Verheijen et al., Bright single-photon sources in bottom-up tailored nanowires, Nat. Commun. 3, 737 (2012).

[19] G. Bulgarini, M. E. Reimer, T. Zehender, M. Hocevar, E. P. A. M. Bakkers, L. P. Kouwenhoven et al., Spontaneous emission control of single quantum dots in bottom-up nanowire waveguides, Appl. Phys. Lett. 100, 121106 (2012).

[20] D. Dalacu, K. Mnaymneh, J. Lapointe, X. Wu, P. J. Poole, G. Bulgarini et al., Ultraclean emission from InAsP quantum dots in defect-free wurtzite InP nanowires, Nano Lett. 12, 5919 (2012).

[21] N. Sköld, M.-E. Pistol, K. A. Dick, C. Pryor, J. B. Wagner, L. S. Karlsson, and L. Samuelson, Microphotoluminescence studies of tunable wurzite InAsP quantum dots embedded in wurzite InP nanowires, Phys. Rev. B 80, 041312(R) (2009). 
[22] J. Heinrich, A. Huggenberger, T. Heindel, S. Reitzenstein, S. Höfling, L. Worschech et al., Single photon emission from positioned GaAs/AlGaAs photonic nanowires, Appl. Phys. Lett. 96, 211117 (2010).

[23] D. Dalacu, K. Mnaymneh, X. Wu, J. Lapointe, G. C. Aers, P. J. Poole et al., Selective-area vapor-liquid-solid growth of tunable InAsP quantum dots in nanowires, Appl. Phys. Lett. 98, 251101 (2011).

[24] M. E. Reimer, D. Dalacu, J. Lapointe, P. J. Poole, D. Kim, G. C. Aers et al., Single electron charging in deterministically positioned InAs/InP quantum dots, Appl. Phys. Lett. 94, 011108 (2009).

[25] D. Dalacu, K. Mnaymneh, V. Sazonova, P. J. Poole, G. C. Aers, J. Lapointe, R. Cheriton, A. J. Spring Thorpe, and R. Williams, Deterministic emitter-cavity coupling using a singlesite controlled quantum dot, Phys. Rev. B 82, 033301 (2010).

[26] C. Schneider, T. Heindel, A. Huggenberger, T. A. Niederstrasser, S. Reitzenstein, A. Forchel et al., Microcavity enhanced single photon emission from an electrically driven site-controlled quantum dot, Appl. Phys. Lett. 100, 091108 (2012).

[27] K. D. Jöns, P. Atkinson, M. Müller, M. Heldmaier, S. M. Ulrich, O. G. Schmidt et al., Triggered indistinguishable single photons with narrow line widths from site-controlled quantum dots, Nano Lett. 13, 126 (2013).

[28] C. Kammerer, G. Cassabois, C. Voisin, M. Perrin, C. Delalande, $\mathrm{Ph}$. Roussignol et al., Interferometric correlation spectroscopy in single quantum dots, Appl. Phys. Lett. 81, 2737 (2002).

[29] V. Zwiller, T. Aichele, and O. Benson, Single-photon Fourier spectroscopy of excitons and biexcitons in single quantum dots, Phys. Rev. B 69, 165307 (2004).

[30] C. Matthiesen, A. N. Vamivakas, and M. Atatüre, Subnatural Linewidth Single Photons from a Quantum Dot, Phys. Rev. Lett. 108, 093602 (2012).

[31] See Supplemental Material at http://link.aps.org/supplemental/ 10.1103/PhysRevB.93.195316 for further experimental details on: the system response of the Michelson interferometer; quantum dot lifetime measurements for dot A and B; polarization and cross-correlation measurements to identify quantum dot emission lines, temperature dependence of the quantum dot emission spectrum, also showing power narrowing at higher temperatures; single-photon interference measurements as a function of excitation energy, including quasi-resonant excitation at the nanowire resonance; as well as theoretical details to extract the inhomogeneous broadening from the HOM interference data and Monte Carlo simulations of power broadening and narrowing for the quantum dot emission spectrum.

[32] J. Claudon, J. Bleuse, N. S. Malik, M. Bazin, P. Jaffrennou, N. Gregersen et al., A highly efficient single-photon source based on a quantum dot in a photonic nanowire, Nat. Photonics 4, 174 (2010).

[33] E. B. Flagg, A. Muller, S. V. Polyakov, A. Ling, A. Migdall, and G. S. Solomon, Interference of Single Photons from Two Separate Semiconductor Quantum Dots, Phys. Rev. Lett. 104, 137401 (2010).

[34] R. B. Patel, A. J. Bennett, I. Farrer, C. A. Nicoll, D. A. Ritchie, and A. J. Shields, Two-photon interference of the emission from electrically tunable remote quantum dots, Nat. Photonics 4, 632 (2010).

[35] P. Gold, A. Thoma, S. Maier, S. Reitzenstein, C. Schneider, S. Höfling, and M. Kamp, Two-photon interference from remote quantum dots with inhomogeneously broadended linewidths, Phys. Rev. B 89, 035313 (2014).

[36] P. Borri, W. Langbein, S. Schneider, U. Woggon, R. L. Sellin, D. Ouyang, and D. Bimberg, Ultralong Dephasing Time in InGaAs Quantum Dots, Phys. Rev. Lett. 87, 157401 (2001).

[37] M. Bayer and A. Forchel, Temperature dependence of the exciton homogeneous linewidth in InGaAs/GaAs self-assembled quantum dots, Phys. Rev. B 65, 041308(R) (2002).

[38] C. Santori, D. Fattal, J. Vuckovic, G. S. Solomon, and Y. Yamamoto, Indistinguishable photons from a single-photon device, Nature (London) 419, 594 (2002).

[39] T. Legero, T. Wilk, M. Hennrich, G. Rempe, and A. Kuhn, Quantum Beat of Two Single Photons, Phys. Rev. Lett. 93, 070503 (2004).

[40] T. Legero, T. Wilk, A. Kuhn, and G. Rempe, Time-resolved two-photon quantum interference, Appl. Phys. B 77, 797 (2003).

[41] A. V. Kuhlmann, J. Houel, A. Ludwig, L. Greuter, D. Reuter, A. D. Wieck et al., Charge noise and spin noise in a semiconductor quantum device, Nat. Phys. 9, 570 (2013).

[42] K. G. Lagoudakis, P. L. McMahon, K. A. Fischer, S. Puri, K. Muller, D. Dalacu et al., Initialization of a spin qubit in a sitecontrolled nanowire quantum dot, New J. Phys. (2016) (to be published).

[43] X. Ding, Y. He, Z.-C. Duan, N. Gregersen, M.-C. Chen, S. Unsleber, S. Maier, C. Schneider, M. Kamp, S. Hofling, C.-Y. Lu, and J.-W. Pan, On-Demand Single Photons with High Extraction Efficiency and Near-Unity Indistinguishability from a Resonantly Driven Quantum Dot in a Micropillar, Phys. Rev. Lett. 116, 020401 (2016).

[44] N. Somaschi, V. Giesz, L. De Santis, J. C. Loredo, M. P. Almeida et al., Near-optimal single-photon sources in the solid state, Nat. Photonics 10, 340 (2016).

[45] A. Thoma et al., Exploring Dephasing of a Solid-State Quantum Emitter via Time- and Temperature-Dependent Hong-Ou-Mandel Experiments, Phys. Rev. Lett. 116, 033601 (2016). 